\title{
A theoretical model of scanning tunneling microscopy: Application to the graphite (0001) and Au(111) surfaces
}

\author{
Hui Ou-Yang, Bruno Källebring, and R. A. Marcus \\ Arthur Amos Noyes Laboratory of Chemical Physics, California Institute of Technology, \\ Pasadena, California $91125^{\text {a) }}$
}

(Received 27 November 1992; accepted 25 January 1993)

\begin{abstract}
An expression for the scanning tunneling microscopy (STM) current between the tip and sample is presented using first-order perturbation theory for a two-Hamiltonian formalism ("reactants" and "products"). The calculated STM current depends on the square of the sample-tip matrix elements, averaged over a selection of random points in wave vector space. In the limit of low voltage and temperature, this averaging is over the Fermi surface of the sample. The model is applied to the graphite (0001) and $\mathrm{Au}(111)$ surfaces using a simple model (chain) of a tungsten tip and the tight-binding approximation. Comparisons with experiments and with the result for graphite obtained by Tersoff and Lang using a molybdenum tip are given. The theory is applied elscwhere to STM of adsorbates.
\end{abstract}

\section{INTRODUCTION}

Scanning tunneling microscopy (STM) has provided a stimulating development in the study of surfaces. ${ }^{1,2}$ It offers the possibility of giving a direct real space image of the surface structure at atomic resolution. In addition to bare surfaces, e.g., Refs. 1 and 2, images of surfaces with adsorbates ranging from single atoms ${ }^{3}$ to molecules of biological interest ${ }^{4}$ have been reported.

One goal of theory is to explain how the STM current in the experiments varies with the tip position, voltage, and, when an adsorbate is present, adsorbate. A variety of theoretical models have been developed to interpret STM experiments and the tunneling mechanism..$^{5-13}$ Since the real tip structure in STM is unknown, a somewhat arbitrary tip model is typically introduced into the theoretical treatments. One of the very first and still widely used models for STM is due to Tersoff and Hamann, ${ }^{5}$ who based their theory on Bardeen's transfer Hamiltonian approach $^{14,15}$ and an $s$-wave tip model. In the limit of low voltage and low temperature, Tersoff and Hamann found that the STM current is proportional to the local density of states (LDOS) of the sample at the position of the tip $\mathbf{r}_{t}{ }^{5}$

$$
I \propto \sum_{s}\left|\Psi_{s}\left(\mathbf{r}_{t}\right)\right|^{2} \delta\left(E_{s}-E_{f}\right)=\text { LDOS },
$$

where $E_{s}$ and $\Psi_{s}$ denote the energy eigenvalue and the wave function of quantum state $s$ of the sample, respectively. Thus, in the constant current mode, the STM image at low voltage represents a contour map of constant LDOS at the Fermi level $E_{f}$. Equation (1) has served as a foundation for many theoretical calculations ${ }^{5,16,17}$ of STM images and has been reported to be a good approximation for simple surfaces, such as $\mathrm{Au}(110) 2 \times 1$ and $3 \times 1$, and $\mathrm{Si}(111)$ $7 \times 7 . .^{18}$ In spite of its success in these systems, ${ }^{18}$ it is recognized that an $s$-wave model oversimplifies the electronic structure of the tip and has been reported by Tersoff and Lang and others to break down for graphite ${ }^{19,20}$ and for close-packed metals surfaces such as $\mathrm{Au}(111)$ and $\mathrm{Al}(111){ }^{21,22}$ Other tip modcls have also been introduced for STM instead of the $s$-wave tip approximation, e.g., in Refs. 6-13.

An aim of the present paper is to develop a model for STM whose formulation is applicable for a wide range of calculational methods, such as density-functional, ${ }^{23-26}$ Hartree-Fock-Slater, ${ }^{27,28}$ and tight-binding, ${ }^{29,30}$ but which still offers a relatively simple interpretation of the STM image. The tip-sample interaction is assumed to be weak in the present treatment. Since the experiments are performcd at room temperature, the low temperature limit for the electronic states of the solid and for the Fermi-Dirac distribution is used throughout.

In the present paper, an expression for the STM current is first deduced using the time-dependent first-order perturbation theory for a two-Hamiltonian system. ${ }^{31}$ The STM current is then related to the interaction matrix elements between sample and tip, which may be treated by density-functional or quantum chemistry calculations, or by the method subsequently used in the present paper, the tight-binding/extended Hückel ${ }^{32}$ approach. For concreteness, the present treatment is next specialized by making use of a simplified tip model in which the tip is assumed to be a semi-infinite linear chain of atoms. The theory is given in Sec. II and applied to the STM images of the graphite (0001) and $\mathrm{Au}(111)$ surfaces in Sec. III. Some comparison with the transfer Hamiltonian approach of Tersoff and Hamann is given in Sec. IV, and some concluding remarks are given in Sec. V.

\section{THEORETICAL MODEL}

\section{A. The STM current between tip and sample}

A one-electron Schrödinger equation is used for the tip and a similar one for the sample (in units of $\hbar=1$ )

$$
\left[-\frac{1}{2 m} \nabla^{2}+v_{\mathrm{eff}}(\mathbf{r})\right] \phi_{n \mathbf{k}}(\mathbf{r})=E_{n}(\mathbf{k}) \phi_{n \mathbf{k}}(\mathbf{r}),
$$

${ }^{2)}$ Contribution No. 8772. 
where $\phi_{n \mathbf{k}}(\mathbf{r})$ and $E_{n}(\mathbf{k})$ are the wave function and the eigenvalue with band index $n$ and wave vector $\mathbf{k}$, respectively, and $v_{\text {eff }}$ is an effective local potential whose explicit form is not immediately needed in the following derivation. A local potential implies that $v_{\text {eff }}(\mathbf{r})$ is the same for different $\phi_{n \mathrm{k}}(\mathbf{r})$ and $E_{n}(\mathbf{k})$. This $v_{\text {eff }}(\mathbf{r})$ can be chosen to be any local potential such as the Kohn-Sham effective potential ${ }^{24}$ in density-functional theory, ${ }^{23-26}$ the potential in HartreeFock-Slater theory, ${ }^{27,28}$ and the potential used in tightbinding ${ }^{29,30}$ or extended Hückel calculations. ${ }^{32}$ We note, on the other hand, that the Hartree-Fock potential itself is not a local potential due to the nonlocality of its exchange part, e.g., Refs. 26 and 27. Symbols $s$ and $t$ are added later to the potentials and to the Hamiltonians, wave functions, and eigenvalues.

We consider an electron moving in a system consisting of the sample and the tip in STM. The Hamiltonian for this electron within any of the local theories mentioned above is given by

$$
H=-\frac{1}{2 m} \nabla^{2}+v_{\mathrm{eff}}^{t}+v_{\mathrm{eff}}^{s}+v_{s-t} \equiv H_{t}+H_{s}+v_{s}{ }_{t}+\frac{1}{2 m} \nabla^{2},
$$

where $H_{s}$ and $H_{t}$, and $v_{\text {eff }}^{s}$ and $v_{\text {eff }}^{t}$ are the Hamiltonians and the effective potentials for the isolated sample and tip, respectively, and $v_{s-t}$ is the sample-tip interaction potential. $H_{t}$ is the sum of the first two terms and $H_{s}$ is the sum of the first and third terms in the first equality in Eq. (3).

In applying time-dependent perturbation theory, the electron at the initial time $t=0$ can bc taken to be localized in the $\phi_{n \mathbf{k}_{t}}(\mathbf{r})$ state of the tip, where $\mathbf{k}_{t}$ denotes the wave vector for the tip states. Similarly, $\mathbf{k}_{s}$ is the wave vector for the sample states. In a three-dimensional treatment of the solid, each $\mathbf{k}_{s}$ represents a running wave parallel to the surface and a standing wave pointing inward from the surface, but denotes only a standing wave in a onedimensional system. When a voltage is applied, we denote by $P_{t \rightarrow s}$ the steady-state transition rate of transfer of an electron from a $\phi_{n \mathbf{k}_{t}}(\mathbf{r})$ state of the tip to a $\phi_{n \mathbf{k}_{s}}(\mathbf{r})$ state of the sample per unit volume of $\mathbf{k}_{s}$ space and per unit volume of $\mathbf{k}_{t}$ space. We denote by $P_{s \rightarrow t}$, the corresponding transition rate for the reverse process. The arguments involve standard time-dependent perturbation theory, but we give them here because of the presence of the two Hamiltonians and the delta function normalization ${ }^{33}$ employed in this paper. ${ }^{34}$ (State counting and units for the transition rate of a process using the present normalization are discussed in Ref. 33.)

For $P_{t \rightarrow s}$, a linear combination of the initial state $\phi_{\mathbf{k}_{t}}(\mathbf{r})$ for the tip and of the final states $\phi_{\mathbf{k}_{s}}(\mathbf{r})$ for the sample is used as the time-dependent wave function ${ }^{31}$

$$
\Psi_{\mathrm{k}}(\mathbf{r}, t)=a_{\mathrm{k}_{t}}(t) \phi_{\mathrm{k}_{t}}(\mathbf{r}) e^{-i E_{t} t}+\int d \mathbf{k}_{s} a_{\mathbf{k}_{s}}(t) \phi_{\mathbf{k}_{s}}(\mathbf{r}) e^{-i E_{s} t}
$$

For notational brevity, $E\left(\mathbf{k}_{i}\right)$ has been written as $E_{i}$, where $i=s$ or $t$, and only a single-band system is considered in Eq.
(4). However, the result is readily generalized to the multiband case, and the final result for the latter is given later in Eq. (14b) below.

Introducing this $\Psi_{k}(\mathbf{r}, t)$ into the time-dependent Schrödinger equation

$$
i \frac{\partial}{\partial t} \Psi_{\mathbf{k}}(\mathbf{r}, t)=\left(\frac{1}{2 m} \nabla^{2}+v_{s-t}+H_{t}+H_{s}\right) \Psi_{\mathbf{k}}(\mathbf{r}, t)
$$

and noting that $E_{i}$ is the eigenvalue of $H_{i}(i=s$ or $t$ ), we obtain, after some rearrangement,

$$
\begin{gathered}
i\left[\dot{a}_{\mathbf{k}_{t}}(t) \phi_{\mathbf{k}_{t}}(\mathbf{r}) e^{-i E_{t} t}+\int d \mathbf{k}_{s} \dot{a}_{\mathbf{k}_{s}}(t) \phi_{\mathbf{k}_{s}}(\mathbf{r}) e^{-i E_{s} t}\right] \\
=a_{\mathbf{k}_{t}}(t)\left(v_{\mathrm{eff}}^{s}+v_{s-t}\right) \phi_{\mathbf{k}_{t}}(\mathbf{r}) e^{-i E_{t} t} \\
\quad+\int d \mathbf{k}_{s} a_{\mathbf{k}_{s}}(t)\left(v_{\mathrm{eff}}^{t}+v_{s-t}\right) \phi_{\mathbf{k}_{s}}(\mathbf{r}) e^{-i E_{s} t}
\end{gathered}
$$

where the dot denotes $d / d t$. Multiplying Eq. (6) by $\phi_{\mathrm{k}_{s}^{\prime}}^{*}(\mathbf{r})$, integrating over all space, and using a Dirac delta function normalization for $\phi_{\mathbf{k}_{s}}(\mathbf{r})$,

$$
\left\langle\phi_{\mathbf{k}_{s}^{\prime}} \mid \phi_{\mathbf{k}_{s}}\right\rangle=\int d \mathbf{r} \phi_{\mathbf{k}_{s}^{\prime}}^{*}(\mathbf{r}) \phi_{\mathbf{k}_{s}}(\mathbf{r})=\delta\left(\mathbf{k}_{s}-\mathbf{k}_{s}^{\prime}\right),
$$

one obtains

$$
\begin{aligned}
i\left[\dot{a}_{\mathbf{k}_{t}}(t)\left\langle\phi_{\mathbf{k}_{s}^{\prime}} \mid \phi_{\mathbf{k}_{t}}\right\rangle e^{\left.-i E_{t^{t}}+\dot{a}_{\mathbf{k}_{s}^{\prime}}(t) e^{-i E_{s^{t}}}\right]}\right. \\
=a_{\mathbf{k}_{t}}(t)\left\langle\phi_{\mathbf{k}_{s}^{\prime}}\left|\left(v_{\mathrm{eff}^{s}}^{s}+v_{s-t}\right)\right| \phi_{\mathbf{k}_{t}}\right\rangle e^{-i E_{t^{t}} t} \\
\quad+\int d \mathbf{k}_{s} a_{\mathbf{k}_{s}}(t)\left\langle\phi_{\mathbf{k}_{s}^{\prime}}\left|\left(v_{\mathrm{eff}}^{t}+v_{s-t}\right)\right| \phi_{\mathbf{k}_{s}}\right\rangle e^{-i E_{s^{\prime}} t}
\end{aligned}
$$

In a first-order approximation, it is assumed as usual that $a_{\mathbf{k}_{t}}(t) \simeq 1$ and $a_{\mathbf{k}_{s}}(t) \simeq 0$ on the right-hand side of Eq. (8). We neglect the overlap integral $\left\langle\phi_{\mathbf{k}_{s}^{\prime}} \mid \phi_{\mathbf{k}_{t}}\right\rangle$, which implies that the electron is assumed to be strongly localized on the tip and on the sample before and after the transition, respectively. Equation (8) then becomes, upon omitting the final primes,

$$
i \dot{a}_{\mathbf{k}_{s}}(t) e^{-i E_{s} t}=\left\langle\phi_{\mathbf{k}_{s}}\left|v_{\mathrm{cff}}^{s}+v_{s-t}\right| \phi_{\mathbf{k}_{t}}\right\rangle e^{-i E_{t} t} .
$$

After integration, one then obtains $P_{t \rightarrow s}$ from $\lim _{t \rightarrow \infty}\left|a_{\mathbf{k}_{s}}\right|^{2} / t$, using a standard argument, with $\lim _{t \rightarrow \infty}\left(\sin ^{2} x t\right) / x^{2} t=\pi \delta(x)$, yielding

$$
\begin{aligned}
P_{t \rightarrow s} & =2 \pi\left|\left\langle\phi_{\mathrm{k}_{s}}\left|v_{\text {eff }}^{s}+v_{s-t}\right| \phi_{\mathrm{k}_{t}}\right\rangle\right|^{2} \delta\left(E_{s}-E_{t}\right) \\
& =2 \pi\left|\left\langle\phi_{\mathbf{k}_{s}}\left|H-H_{t}\right| \phi_{\mathrm{k}_{t}}\right\rangle\right|^{2} \delta\left(E_{s}-E_{t}\right) \\
& \simeq 2 \pi\left|H\left(\mathbf{k}_{s}, \mathbf{k}_{t}\right)\right|^{2} \delta\left(E_{s}-E_{t}\right)
\end{aligned}
$$

after using $H_{t} \phi_{\mathbf{k}_{t}}=E_{t} \phi_{\mathbf{k}_{t}}$ and then again neglecting the overlap $\left\langle\phi_{\mathbf{k}_{s}} \mid \phi_{\mathrm{k}_{t}}\right\rangle$. In Eq. (10), $E_{s}$ and $E_{t}$ are the energies of states of the sample and tip, respectively, ${ }^{35}$ and $\left|H\left(\mathbf{k}_{s}, \mathbf{k}_{t}\right)\right|^{2}$ denotes $\left|\left\langle\phi_{\mathrm{k}_{s}}|H| \phi_{\mathrm{k}_{t}}\right\rangle\right|^{2}$.

It is useful to rewrite $E_{s}$ and $E_{t}$ in terms of values relative to the respective Fermi levels (the chemical potentials) $\mu_{s}$ and $\mu_{t}$ of the two solids 


$$
\epsilon_{s}=E_{s}-\mu_{s}, \quad \epsilon_{t}=E_{t}-\mu_{t},
$$

and to note that the net current vanishes when $\mu_{s}=\mu_{t}$. The quantities $E_{s}, E_{t}, \mu_{s}$, and $\mu_{t}$ are all linearly dependent on the respective potentials acting on $s$ and on $t$, and so we may also write ${ }^{36}$

$$
\mu_{s}-\mu_{t}=e v
$$

where $v$ is the potential of the tip minus that of the sample. ${ }^{36}$ Accordingly, we have $\delta\left(E_{s}-E_{l}\right)=\delta\left(\epsilon_{s}-\epsilon_{t}+e v\right)$.

The electronic current from tip to sample is given by Eq. (10), multiplied by the probability $f\left(\epsilon_{t}\right)$ that the tip level $t$ is occupied, and by $\left[1-f\left(\epsilon_{s}\right)\right]$, the probability that the sample level $s$ is unoccupied, and integrated over all $\mathbf{k}_{s}$ and $\mathbf{k}_{t}$ (by virtue of the units of $\phi_{\mathrm{k}_{s}}$ and $\left.\phi_{\mathrm{k}_{t}}\right) .{ }^{33}$ Here $f(\epsilon)$ is the Fermi-Dirac distribution function $[1+\exp (\epsilon)$ $\left.\left.k_{B} T\right)\right]^{-1}$, with $k_{B}$ and $T$ being the Boltzmann constant and absolute temperature, respectively. We then obtain

$$
\begin{gathered}
I_{\{t\} \rightarrow\{s\}}=2 \pi e \iint d \mathbf{k}_{s} d \mathbf{k}_{t}\left|H\left(\mathbf{k}_{s}, \mathbf{k}_{t}\right)\right|^{2} f\left(\epsilon_{t}\right) \\
\times\left[1-f\left(\epsilon_{s}\right)\right] \delta\left(\epsilon_{s}-\epsilon_{t}+e v\right) .
\end{gathered}
$$

Similarly, we have

$$
\begin{aligned}
I_{\{s\} \rightarrow\{t\}}= & 2 \pi e \iint d \mathbf{k}_{s} d \mathbf{k}_{t}\left|H\left(\mathbf{k}_{s}, \mathbf{k}_{t}\right)\right|^{2} f\left(\epsilon_{s}\right) \\
& \times\left[1-f\left(\epsilon_{t}\right)\right] \delta\left(\epsilon_{s}-\epsilon_{t}+e v\right) .
\end{aligned}
$$

The net current from tip to sample is then given by

$$
\begin{aligned}
I= & I_{\{t\} \rightarrow\{s\}}-I_{\{s\} \rightarrow\{t\}} \\
= & 2 \pi e \iint d \mathbf{k}_{s} d \mathbf{k}_{t}\left|H\left(\mathbf{k}_{s} \mathbf{k}_{t}\right)\right|^{2}\left[f\left(\epsilon_{t}\right)-f\left(\epsilon_{s}\right)\right] \\
& \times \delta\left(\epsilon_{s}-\epsilon_{t}+e v\right) .
\end{aligned}
$$

In the multiband case, treating the bands as prediagonalized, the Hamiltonian matrix element $\left\langle\phi_{n \mathbf{k}_{s}}|H| \phi_{m \mathbf{k}_{t}}\right\rangle$ now depends on $n$ and $m$, the band indices for the sample and tip, respectively. If this matrix element is denoted by $H_{n m}\left(\mathbf{k}_{s}, \mathbf{k}_{t}\right)$, one sums Eqs. (13a) and (13b) over $m$ and $n$ and, instead of Eq. (14a), obtains

$$
\begin{aligned}
I= & 2 \pi e \sum_{n, m} \iint d \mathbf{k}_{s} d \mathbf{k}_{t}\left|H_{n m}\left(\mathbf{k}_{s}, \mathbf{k}_{t}\right)\right|^{2}\left[f\left(\epsilon_{t}\right)-f\left(\epsilon_{s}\right)\right] \\
& \times \delta\left(\epsilon_{s}-\epsilon_{t}+e v\right) .
\end{aligned}
$$

In the limit of low voltage $v$, Eqs. (14a) and (14b) reduce to

$$
\begin{aligned}
I & =I_{\{t\} \rightarrow\{s\}}-I_{\{s\} \rightarrow\{t\}} \\
& =2 \pi e^{2} v \iint d \mathbf{k}_{s} d \mathbf{k}_{t}\left|H\left(\mathbf{k}_{s} \mathbf{k}_{t}\right)\right|^{2} \delta\left(\epsilon_{s}\right) \delta\left(\epsilon_{t}\right)
\end{aligned}
$$

and

$$
I=2 \pi e^{2} v \sum_{n, m} \iint d \mathbf{k}_{s} d \mathbf{k}_{t}\left|H_{n m}\left(\mathbf{k}_{s}, \mathbf{k}_{t}\right)\right|^{2} \delta\left(\epsilon_{s}\right) \delta\left(\epsilon_{t}\right)
$$

respectively. In the present paper, we shall be only concerned with this limiting case, but in later work the full formulas (14a) and (14b) will be used to study the effect of $v$. Using a specific tip model, it is next shown here how $\left|H\left(\mathbf{k}_{s}, \mathbf{k}_{t}\right)\right|^{2}$ or $\left|H_{n m}\left(\mathbf{k}_{s}, \mathbf{k}_{t}\right)\right|^{2}$ can be simplified.

\section{B. A tip model-semi-infinite chain of atoms}

We make use of a simple tip model in which the tip is assumed to be a semi-infinite linear chain of atoms. A similar tip model was used by Sacks and Noguera. ${ }^{13}$ Ultimately, one would wish to introduce, for comparison, tip models with other geometrical shapes. For simplicity of presentation, we consider a chain with one electron per atom and one orbital per atom, the hopping integral $H_{i j}$ between nearest neighbors being denoted by $\beta$, and $H_{i i}$ by $\alpha$. The tight-binding approximation will be used neglecting the overlap integrals for simplicity and retaining only the Hamiltonian matrix elements between nearest neighbors. For this model, $\epsilon_{t}\left(\mathbf{k}_{t}\right)$ defined in Eq. (11) and $\phi_{\mathbf{k}_{t}}(\mathbf{r}) \operatorname{are}^{37}$

$$
\begin{aligned}
& \epsilon_{t}\left(\mathbf{k}_{t}\right)=\alpha-\mu_{t}+2 \beta \cos 2 \pi k_{t}=2 \beta \cos 2 \pi k_{t} \\
& \phi_{\mathbf{k}_{t}}(\mathbf{r})=\sqrt{2} \sum_{l=1}^{\infty} \sin 2 \pi l k_{t} \phi_{l}(z)
\end{aligned}
$$

where $z$ is the linear coordinate along the chain; $\phi_{l}(z)$ denotes the atomic orbital on site $l$; and $\phi_{\mathbf{k}_{t}}(\mathbf{r}), \phi_{k_{t}}(z)$ being normalized via $\left\langle\phi_{k_{t}} \mid \phi_{k_{t}^{\prime}}\right\rangle=\delta\left(k_{t}-k_{t}^{\prime}\right), 0<k_{t}<\frac{1}{2}$. For this model, as noted in the second equality in Eq. (16a), $\mu_{t}$ equals $\alpha$, since the Fermi level occurs at the middle of the single band system where there is, per atom, one electron and one orbital.

Introducing Eqs. (16) for $\epsilon_{t}\left(k_{t}\right)$ and $\phi_{k_{t}}(z)$ into Eq. (15b), we have

$$
\begin{aligned}
I= & 4 \pi e^{2} v \sum_{n} \int d \mathbf{k}_{s} \int_{0}^{1 / 2} d k_{t}\left|\left\langle\phi_{l}|H| \phi_{n \mathbf{k}_{s}}\right\rangle\right|^{2} \delta\left(\epsilon_{s}\right) \\
& \times \delta\left(\epsilon_{t}\right) \sin ^{2} 2 \pi k_{t}
\end{aligned}
$$

where, as an approximation, only the matrix element between the first tip atom [orbital $\phi_{t}$ is used to denote the $\phi_{1}$ in Eq. (16b)] and the sample has been included. We next note that

$$
\begin{gathered}
4 \pi|\beta| \int_{0}^{1 / 2} d k_{t} \sin ^{2} 2 \pi k_{t} \delta\left(\epsilon_{t}\right) \\
\quad=\int_{-1}^{1} d\left(\cos 2 \pi k_{t}\right) \sqrt{\left(1-\cos ^{2} 2 \pi k_{t}\right)} \delta\left(\cos 2 \pi k_{t}\right)=1
\end{gathered}
$$




$$
\begin{aligned}
I & =\frac{e^{2} v}{|\beta|} \sum_{n} \int d \mathbf{k}_{s}\left|\left\langle\phi_{t}|H| \phi_{n \mathbf{k}_{s}}\right\rangle\right|^{2} \delta\left(\epsilon_{s}\right) \\
& \equiv \frac{e^{2} v}{|\beta|} \sum_{n} \int d \mathbf{k}_{s}\left|H_{t, n \mathbf{k}_{s}}\right|^{2} \delta\left(\epsilon_{s}\right) \\
& \propto \rho_{s}^{0}\left\langle\left|H_{t, n \mathbf{k}_{s}}\right|^{2}\right\rangle_{\mathrm{av}},
\end{aligned}
$$

where $\rho_{s}^{0}$ denotes the sample density of states at the Fermi energy $\epsilon_{s}=0$ with $\rho_{s}(\epsilon)$ being defined as $\rho_{s}(\epsilon)$ $=\Sigma_{n} \int d \mathbf{k}_{s} \delta\left(\epsilon-\epsilon_{s}\right)$ and where $\left\langle\left|H_{t, n \mathbf{k}_{s}}\right|^{2}\right\rangle_{\text {av }}$ denotes the average over the Fermi surface

$$
\begin{aligned}
\left\langle\left|H_{t, n \mathbf{k}_{s}}\right|^{2}\right\rangle_{\mathrm{av}}= & \sum_{n} \int d \mathbf{k}_{s}\left|H_{t, n \mathbf{k}_{s}}\right|^{2} \\
& \times \delta\left(\epsilon_{s}\right) / \sum_{n} \int d \mathbf{k}_{s} \delta\left(\epsilon_{s}\right) .
\end{aligned}
$$

It is seen from Eq. (19a) that the STM current in this model is simply proportional to $\left\langle\left|H_{t, n \mathbf{k}_{s}}\right|^{2}\right\rangle_{\mathrm{av}}$ the squared electronic matrix element averaged over a selection of random points in wave vector space on the Fermi surface of the sample. The STM image is then simply a contour of constant $\left\langle\left|H_{t, n \mathbf{k}_{s}}\right|^{2}\right\rangle_{\mathrm{av}}$.

We next consider a tip consisting of transition metal atoms in which the $d$ electrons (bands) dominate the properties, including the Fermi surface. It can be readily shown that for a semi-infinite linear chain of transition metal atoms, the five $d$ orbitals $d_{z^{2}}, d_{x^{2}-y^{2}} d_{x y y}, d_{y z}$ and $d_{x z}$ do not interact with each other. ${ }^{38}$ We then have five noninteracting $d$ bands whose energies and wave functions are given by

$$
\begin{aligned}
& \epsilon_{m}\left(\mathbf{k}_{t}\right)=\alpha_{m}-\mu_{t}+2 \beta_{m} \cos 2 \pi k_{t} \\
& \phi_{m \mathbf{k}_{t}}(\mathbf{r})=\sqrt{2} \sum_{j=1}^{\infty} \sin 2 \pi j k_{t} d_{m}^{j},
\end{aligned}
$$

where $d_{m}^{j}$ denotes the $m$ th $d$ orbital on the site $j$ and where the parameters $\alpha_{m}$ and $\beta_{m}$ are defined as $\alpha_{m}=\left\langle d_{m}^{j}|H| d_{m}^{i}\right\rangle$ and $\beta_{m}=\left\langle d_{m}^{j}|H| d_{m}^{j \pm 1}\right\rangle$, respectively. In the extended Hückel calculation, five $\alpha_{m}$ 's are approximated to be the same and $\alpha$ will then be used in place of $\alpha_{m}$ in the following.

Introducing Eqs. (20a) and (20b) for $\epsilon_{m}\left(k_{t}\right)$ and $\phi_{m k_{t}}(\mathbf{r})$ into Eq. (15b), we have

$$
\begin{aligned}
I= & 4 \pi e^{2} v \sum_{n} \sum_{m} \int d \mathbf{k}_{s} \int_{0}^{1 / 2} d k_{t}\left|\left\langle d_{m}^{t}|H| \phi_{n \mathbf{k}_{s}}\right\rangle\right|^{2} \\
& \times \sin ^{2} 2 \pi k_{t} \delta\left(\alpha-\mu_{t}+2 \beta_{m} \cos 2 \pi k_{t}\right) \delta\left(\epsilon_{s}\right)
\end{aligned}
$$

where $d_{m}^{t}$ denotes the $m$ th $d$ orbital of the first tip atom (the atom closest to the sample). Here again, as an approximation, only the matrix elements between the sample and the atomic orbitals $d_{m}^{t}$ of the first tip atom have been included. Using

$$
\begin{gathered}
2 \pi \int_{0}^{1 / 2} d k_{t} \sin ^{2} 2 \pi k_{t} \delta\left(\alpha-\mu_{t}+2 \beta_{m} \cos 2 \pi k_{t}\right) \\
=\left[\left(2 \beta_{m}\right)^{2}-\left(\mu_{t}-\alpha\right)^{2}\right]^{1 / 2} /\left(2 \beta_{m}\right)^{2}
\end{gathered}
$$

Eq. (21) for $I$ becomes

$$
\begin{aligned}
I= & 2 e^{2} v \sum_{n} \sum_{m} \int d \mathbf{k}_{s}\left[\left(2 \beta_{m}\right)^{2}-\left(\mu_{t}-\alpha\right)^{2}\right]^{1 / 2} \\
& \times\left|\left\langle d_{m}^{t}|H| \phi_{n \mathbf{k}_{s}}\right\rangle\right|^{2} \delta\left(\epsilon_{s}\right) /\left(2 \beta_{m}\right)^{2} .
\end{aligned}
$$

When $\beta_{m}$ is approximated to be independent of $m$ and is then denoted by $\bar{\beta}$, we have

$$
\begin{aligned}
I= & 2 e^{2} v\left\{\left[(2 \bar{\beta})^{2}-\left(\mu_{t}-\alpha\right)^{2}\right]^{1 / 2} /(2 \bar{\beta})^{2}\right\} \\
& \times \sum_{n} \int d \mathbf{k}_{s}\left|H_{t, n \mathbf{k}_{s}}\right|^{2} \delta\left(\epsilon_{s}\right) \\
\propto & \rho_{s}^{0}\left\langle\left|H_{t, n \mathbf{k}_{s}}\right|^{2}\right\rangle_{\mathrm{av}},
\end{aligned}
$$

where $\left|H_{t, n \mathbf{k}_{s}}\right|^{2}$ denotes

$$
\left|H_{t, n \mathbf{k}_{s}}\right|^{2}=\sum_{m=1}^{5}\left|\left\langle d_{m}^{t}|H| \phi_{n \mathbf{k}_{s}}\right\rangle\right|^{2} .
$$

Here again, $\rho_{s}^{0}$ denotes the sample density of states at the Fermi energy $\epsilon_{s}=0$ and $\left\langle\left|H_{t, n \mathbf{k}_{s}}\right|^{2}\right\rangle_{\text {av }}$ is defined in Eq. (19b). Some features of the formalism are next illustrated by treating bare graphite and gold, where previous results are available for comparison.

\section{RESULTS}

\section{A. Graphite (0001) surface}

Despite extensive studies of graphite, ${ }^{16,19,20}$ the present knowledge of the corrugation and the asymmetry between $\alpha$ and $\beta$ sites (atoms) is still limited, with the $\alpha$ sites having carbon atoms directly below them and the $\beta$ sites having no atoms immediately below. The corrugation is defined to be the difference between the maximum and minimum tip heights in the constant current mode, and the asymmetry is the difference in the current in the constant height mode or the tip heights in the constant current mode between adjacent sites. In this section, calculations for the corrugation of graphite in the constant current mode are performed, using the theoretical model described in the preceding section. The wave functions and other properties of graphite needed in the calculations were obtained in a previous paper. ${ }^{37}$ For comparison, both the tungsten and hydrogen tips are used. In the following, we first derive expressions for $\left|H_{t, n \mathbf{k}_{s}}\right|^{2}$ in Eqs. (19a) and (23b), a key quantity in calculation of the STM images, and then give the calculated results in the constant current mode. by $^{37}$

For graphite a tight-binding result for $\phi_{n \mathbf{k}_{s}}(\mathbf{r})$ is given 


$$
\begin{aligned}
\phi_{n \mathbf{k}_{s}}(\mathbf{r})= & \sum_{j=\alpha, \beta} A_{n j} \sum_{l_{3}=1,3,5}^{\infty} \sum_{l_{1}, l_{2}} \sin 2 \pi k_{3} l_{3} e^{i \mathbf{k}_{\|} \cdot \mathbf{r}_{l}^{i}} p_{z}\left(\mathbf{r}-\mathbf{r}_{l}^{i}\right) \\
& +\sum_{i=\alpha^{\prime}, \beta^{\prime}} A_{n i} \sum_{l_{3}=2,4,6}^{\infty} \sum_{l_{1}, l_{2}} \sin 2 \pi k_{3} l_{3} e^{i \mathbf{k}_{\|} \cdot \mathbf{r}_{l}^{i}} \\
& \times p_{z}\left(\mathbf{r}-\mathbf{r}_{l}^{i}\right), \quad\left(j=\alpha, \beta ; i=\alpha^{\prime}, \beta^{\prime}\right)
\end{aligned}
$$

where $\mathbf{k}_{s}=\mathbf{k}_{\|}+k_{3} \mathbf{b}_{3}, \mathbf{k}_{\|}=k_{1} \mathbf{b}_{1}+k_{2} \mathbf{b}_{2}$, and $\mathbf{b}_{i}$ denotes a reciprocal lattice vector. ${ }^{49} \mathbf{k}_{\|}$represents the twodimensional wave vector parallcl to the surface $\left(-\frac{1}{2} \leqslant k_{1}\right.$, $k_{2} \leqslant \frac{1}{2} ; 0<k_{3}<\frac{1}{2}$ ). In Eq. (25), $\mathrm{r} g$ is the lattice vector for $g$ type atoms $g=\alpha, \beta, \alpha^{\prime}$, and $\beta^{\prime}$, and the subscript $l$ denotes the triad $\left(l_{1} l_{2} l_{3}\right) \cdot{ }^{37} p_{z}\left(\mathbf{r}-\mathbf{r}^{g}\right)$ is the $p_{z}$ wave function centered at $\mathbf{r}=\mathbf{r}^{2}$. The approximations used to obtain Eq. (25) for $\phi_{n k_{s}}(r)$ and to determine the coefficients $A_{n g}$ are given in detail in Ref. $37 .^{50}$ We consider a hydrogen tip (a semiinfinite chain of hydrogen atoms) first.

The distance between two graphite layers is $3.35 \AA$ (Ref. 51) and it is thus natural to neglect the interactions between the tip atoms with the second and further layers of graphite. The interactions due to the second and further atoms of the tip are also ignored. The matrix element $H_{l, n \mathrm{k}_{s}}$ in Eq. (19a) can then be written as

$H_{t, n \mathrm{k}_{s}}=\left\langle s_{t}|H| \phi_{n \mathrm{k}_{s}}\right\rangle=\sum_{j} A_{n j} \sum_{l} \sin 2 \pi k_{3} e^{i \mathrm{k}_{\|}} \cdot \mathrm{r}_{l}^{j}\left\langle s_{t}|H| p_{z}^{\mathrm{r}_{l}}\right\rangle$,

where $s_{t}$ denotes the $s$ orbital of the first hydrogen atom of the semi-infinite chain tip. Writing $A_{n j}=a_{n j}+i b_{n j}, j=\alpha, \beta$, we have

$$
\begin{aligned}
H_{t, n \mathbf{k}_{s}}= & \sum_{j} \sum_{l} \sin 2 \pi k_{3}\left[\left(a_{n j} \cos \mathbf{k}_{\|} \cdot \mathbf{r}_{l}^{j}-b_{n j} \sin \mathbf{k}_{\|} \cdot \mathbf{r}_{l}^{j}\right)\right. \\
& \left.-i\left(b_{n j} \cos \mathbf{k}_{\|} \cdot \mathbf{r}_{l}^{j}+a_{n j} \sin \mathbf{k}_{\|} \cdot \mathbf{r}_{l}^{j}\right)\right]\left\langle s_{t}|H| p_{z}^{\mathbf{r}_{l}^{j}}\right\rangle,
\end{aligned}
$$

where the coefficients $a_{n j}$ and $b_{n j}$ are determined by the method discussed in Ref. 37 . We then obtain the expression for $\left|H_{t, n \mathbf{k}_{s}}\right|^{2}$,

$$
\begin{aligned}
\left|H_{t, n \mathbf{k}_{s}}\right|^{2}= & \sum_{j, j^{\prime}} \sum_{l, l^{\prime}}\left(\sin 2 \pi k_{3}\right)^{2}\left\langle s_{t}|H| p_{z}^{\mathbf{r}_{l}^{j}}\right\rangle\left\langle p_{z}^{j_{l^{\prime}}^{j^{\prime}}}|H| s_{t}\right\rangle \\
& \times\left[\left(a_{n j} a_{n j^{\prime}}+b_{n j} b_{n j^{\prime}}\right) \cos \mathbf{k}_{\|} \cdot\left(\mathbf{r}_{l^{\prime}}^{j^{\prime}}-\mathbf{r}_{l}^{j}\right)\right. \\
& \left.-\left(a_{n j} b_{n j^{\prime}}-a_{n j^{\prime}} b_{n j}\right) \sin \mathbf{k}_{\|} \cdot\left(\mathbf{r}_{l^{\prime}}^{j^{\prime}}-\mathbf{r}_{l}^{j}\right)\right]
\end{aligned}
$$

We next consider a tungsten tip where $d$ orbitals dominate at the Fermi surface. A simplified expression for $\left|H_{t, n k_{s}}\right|^{2}$ in Eqs. (23b) and (24) for the tungsten tip can then be written as

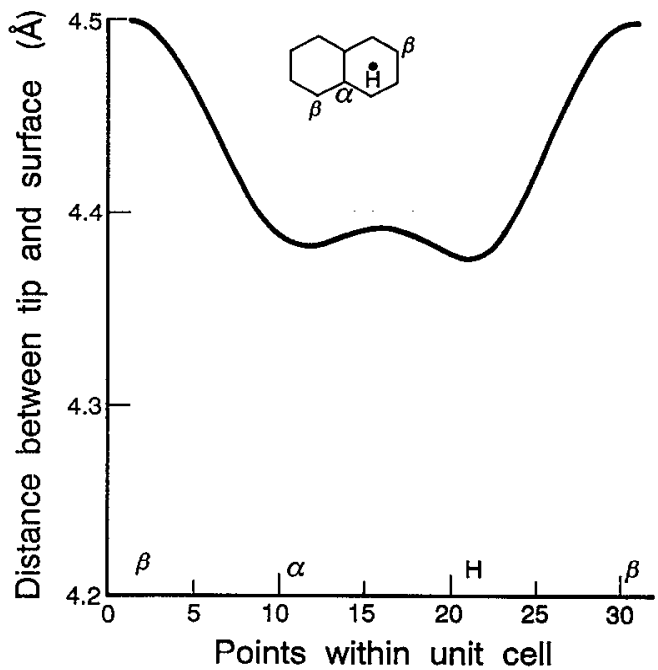

FIG. 1. Calculated scan for graphite with a tungsten tip. (The geometry and other properties of graphite are given in Ref. 38.)

$$
\begin{aligned}
\left|H_{t, n \mathbf{k}_{s}}\right|^{2}= & \sum_{m} \sum_{j, j^{\prime}} \sum_{l, l^{\prime}}\left(\sin 2 \pi k_{3}\right)^{2}\left\langle d_{m}^{t}|H| p_{z}^{\mathbf{r}_{l}^{\prime}}\right\rangle\left\langle p_{z}^{\mathbf{r}_{l^{\prime}}^{j^{\prime}}}|H| d_{m}^{t}\right\rangle \\
& \times\left[\left(a_{n j} a_{n j^{\prime}}+b_{n j} b_{n j^{\prime}}\right) \cos \mathbf{k}_{\|} \cdot\left(\mathbf{r}_{l^{\prime}}^{j^{\prime}}-\mathbf{r}_{l}^{j}\right)\right. \\
& \left.-\left(a_{n j} b_{n j^{\prime}}-a_{n j^{\prime}} b_{n j}\right) \sin \mathbf{k}_{\|} \cdot\left(\mathbf{r}_{l^{\prime}}^{j^{\prime}}-\mathbf{r}_{l}^{j}\right)\right]
\end{aligned}
$$

where $m$ sums over the five $d$ orbitals, the superscript $t$ indicating the first atom of the tip. According to Eqs. (19a) and (23b), in the constant current mode, the STM image follows a contour of constant $\left\langle\left|H_{t, n \mathbf{k}_{s}}\right|^{2}\right\rangle_{\mathrm{av}}$.

The calculational procedure for obtaining $a_{n j}, b_{n j}$, and the $k$ points in Eqs. (27a) and (27b) is described in Ref. 37. In the present calculations of $\left\langle\left|H_{t, n \mathbf{k}_{s}}\right|^{2}\right\rangle_{\mathrm{av}}, 60 \mathrm{k}$ points are randomly chosen from the Fermi surface of graphite. The calculated STM scans for graphite with a tungsten tip and a hydrogen tip are shown in Figs. 1 and 2, respectively.



FIG. 2. Calculated scan for graphite with a hydrogen tip. 
In both cases, the tip is moved from the $\beta$ site through the $\alpha$ and the $H$ (hollow) sites, and then back to the $\beta$ site, where the $H$ site is the point in the middle of the hexagon. In both calculations, the tip is initially placed $4.5 \AA$ above the $\beta$ site, at which $\left\langle\left|H_{t, n k_{s}}\right|^{2}\right\rangle_{\text {av }}$ is evaluated, and the value of that averaged matrix element is then fixed. In order to achieve constancy of $\left\langle\left|H_{t, n \mathbf{k}_{s}}\right|^{2}\right\rangle_{\text {av }}$ over other sites such as the $\alpha$ and $H$ along the line, the tip is moved up or down in the direction perpendicular to the graphite surface (the $z$ direction).

In the above way, corrugations of slightly over 0.1 and of about $2 \AA$ are found for the tungsten and hydrogen tips, respectively. The small corrugation for the tungsten tip is similar to that obtained by Tersoff and Lang, who estimated a corrugation of about $0.1 \AA$ for the Mo tip, smaller than the values reportedly seen in any experiments. ${ }^{19}$

The Tersoff-Lang calculation appeared to be the only one going both beyond the $s$-wave model and addressing the corrugation problem. Experimentally, conclusive values of the corrugation and asymmetry have not yet been determined, and the tip dependence of these properties has not been investigated systematically. The reported experimental corrugation of graphite can be unphysically as large as $24 \AA .{ }^{46}$ Abnormally high corrugation has been postulated to be related to the surface deformation and contamination. ${ }^{46} \mathrm{~A}$ smaller corrugation of about $1 \AA$ has been reported in vacuum. ${ }^{39,42}$ However, Tersoff and Lang have argued that even the $1 \AA$ corrugation includes substantial enhancement from mechanical interactions, which are not fully understood. ${ }^{19}$ In that case, the experimental corrugation that purely reflects the electronic interaction between the tip and sample is still unknown.

The behavior of the $\alpha$ and $\beta$ sites of graphite is of interest. One explanation for the asymmetry of the STM images of graphite is provided by Tomanek et al., who argued that the wave functions of graphite at the Fermi level consist mainly of the $2 p_{z}$ states of the $\beta$ atoms, so that $\beta$ atoms instead of $\alpha$ atoms show up in the STM images. ${ }^{46,52} \mathrm{~A}$ tip dependence of the asymmetry, i.e., of the different behavior of $\alpha$ and $\beta$ sites, was calculated by Tsukada and co-workers, using various clusters to model the tip. ${ }^{48}$ The present calculations indicate an additional feature on the tip dependence of the asymmetry between the $\alpha$ and $\beta$ sites and of the difference in tip positions between the $\alpha$ and $H$ sites. The positions of $\alpha$ and $H$ are depicted in Figs. 1 and 2. In Figs. 1 and 2, the tip positions over the $\alpha$ and $H$ sites are about the same for a tungsten tip, but the tip position is substantially higher over the $\alpha$ site than that over the $H$ site for a hydrogen tip. The difference in the tip heights between $\alpha$ and $\beta$, or equivalently the asymmetry between $\alpha$ and $\beta$, also increases from the tungsten to hydrogen tips, as already noted. The small calculated corrugation of the STM images of graphite of about $0.1 \AA$ using a tungsten tip (Fig. 1) contrasts markedly with that of about $2 \AA$ using a hydrogen tip. In the present model, the STM current is tip dependent through $\left|H_{t, n \mathbf{k}_{s}}\right|^{2}$. As a result, all the quantities such as the corru- gation and the asymmetry related to the current are also tip dependent.

A picture of these variations may be qualitatively given as follows involving the wave functions of graphite on the Fermi surface: These wave functions occupied at the Fermi energy are composed chiefly of the $p_{z}$ orbitals localized at the $\beta$ sites, though a small portion of the $p_{z}$ orbitals at the $\alpha$ sites are also occupied at the Fermi energy. The $1 s$ orbital of a hydrogen atom is well localized around its nucleus and its interaction with the $H$ site of the graphite surface is weak because there is no localized atomic orbital at the $H$ site. This interaction increases as the tip moves from the $H$ site to the $\alpha$ and to the $\beta$ sites. For a tungsten tip, the $d$ orbitals are more diffuse. Even when the tip is over the $H$ site, these $d$ orbitals still interact well with the $p_{z}$ orbitals at the $\beta$ and $\alpha$ sites (there are three nearest $\beta$ and $\alpha$ sites in each case). When the tip is over an $\alpha$ site, the $d$ orbitals couple with three dominant $\beta$ orbitals, as well as with one $\alpha$ orbital below with a small coefficient. It appears from the calculations that for the tungsten tip, the $\alpha$ site is almost the same as the $H$ site in terms of their couplings with the tip as in Fig. 1. It is clear that among the $H, \alpha$, and $\beta$ sites, the calculated differences in interactions with the tip are much less for a tungsten tip than for a hydrogen tip, so there is less corrugation, as seen on comparing Figs. 1 and 2 .

\section{B. Au(111) surface}

Atomically resolved images of closed-packed metal surfaces such as $\mathrm{Au}(111)$ (Ref. 53) and $\mathrm{Al}(111)$ (Ref. 54) have been obtained with STM, and tungsten (W) tips were used in those experiments. It was noted that these images cannot be explained by the $s$-wave tip model. ${ }^{21,22}$ Calculations for the corrugation of the $\mathrm{Al}(111)$ surface, using more detailed tip models, were also reported, and the calculated corrugations are in reasonable agreement with the experimental values. ${ }^{21,22}$ In the present section, the corrugation is calculated for the $\mathrm{Au}(111)$ surface, using the present model and a W tip consisting of the semi-infinite linear chain of $\mathrm{W}$ atoms.

It is recalled that in the limit of low voltage, only the wave functions at the Fermi level contribute to calculation of the relevant $\left|H_{t, n \mathbf{k}_{s}}\right|^{2}$. Determination of the Fermi surface of bulk Au has demonstrated that the $s$ band dominates the wave functions at the Fermi level and that the $d$ bands lie at energies lower than the Fermi energy. ${ }^{55}$ As an approximation, we consider only the $s$ band in the calculation of the wave functions for a semi-infinite $\mathrm{Au}$ with the (111) surface. In this case, the wave functions are given by $^{37}$

$$
\begin{aligned}
\phi_{\mathrm{Au}} \equiv \phi_{n=1 \mathbf{k}_{s}}(\mathbf{r})= & \sqrt{2} \sum_{l_{1}, l_{2}=-\infty}^{\infty} \sum_{l_{3}=1}^{\infty}(|F| / F)^{l_{3}} \sin 2 \pi k_{3} l_{3} \\
& \times \exp \left(i \mathbf{k}_{\|} \cdot \mathbf{r}_{l}\right) s\left(\mathbf{r}-\mathbf{r}_{l}\right)
\end{aligned}
$$

where $F$ is defined as

$$
F=\beta\left[\exp \left(i 2 \pi k_{1}\right)+\exp \left(i 2 \pi k_{2}\right)+1\right],
$$




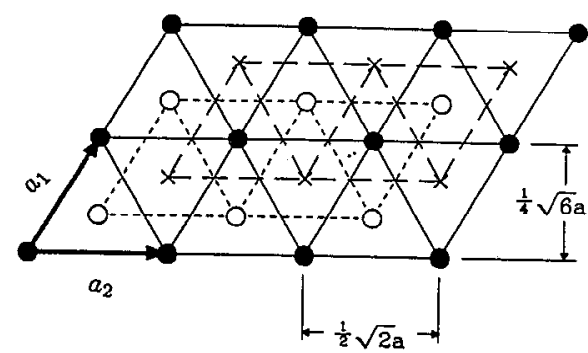

FIG. 3. The geometry of the Au (111) surface. Solid circles are located on $l_{3}$ th layer, open circles on $\left(l_{3}+1\right)$ th layer, and crosses on $\left(l_{3}-1\right)$ th layer. The side of a cubic unit cell, $a$, is $4.08 \AA$, and the spacing between layers is $a / \sqrt{3}$.

with $\beta$ being the hopping integral between two neighboring $s$ orbitals, and using the earlier definition of $\mathbf{k}_{s}$ in terms of the reciprocal lattice vector coordinates $\left(k_{1}, k_{2}, k_{3}\right)$. Also, $\mathbf{r}_{l}=\Sigma_{i=1}^{3} l_{i} \mathbf{a}_{i}$, the subscript $l$ denoting the triad $\left(l_{1} l_{2} l_{3}\right)$ and $\mathbf{a}_{i}$ denoting a lattice vector. A more detailed discussion of Eqs. (28) and (29) is given in Ref. 37. $\left|H_{t, n k_{s}}\right|^{2}$ can now be explicitly written as

$$
\begin{aligned}
\left|H_{t, n \mathbf{k}_{s}}\right|^{2}= & \sum_{l, l^{\prime}} \sum_{m}\left(\sin 2 \pi k_{3}\right)^{2}\left\langle s_{\mathbf{r}_{l}}|H| d_{m}^{t}\right\rangle\left\langle d_{m}^{t}|H| s_{\mathbf{r}_{l^{\prime}}}\right\rangle \\
& \times \cos \mathbf{k}_{\|} \cdot\left(\mathbf{r}_{l^{\prime}}-\mathbf{r}_{l}\right),
\end{aligned}
$$

where only the interactions between the first $\mathrm{W}$ tip atom and atoms within the first layer of the $\mathrm{Au}$ (111) surface are included, and where $m$ denotes the five $d$ orbitals of the $\mathrm{W}$ atom. In the calculations of $\left\langle\left|H_{t, n \mathrm{k}_{s}}\right|^{2}\right\rangle_{\mathrm{av}} 60 \mathrm{k}\left(k_{1}, k_{2}, k_{3}\right)$ points were randomly chosen from the Fermi surface for a semi-infinite Au metal with a (111) surface.

The geometry of a closed-packed Au(111) surface is depicted in Fig. 3. The tip positions, along the path ASAH in Fig. 3 and corresponding to a constant $\left\langle\left|H_{t, n \mathbf{k}_{s}}\right|^{2}\right\rangle_{\text {av }}$, are shown in Fig. 4. The tip starts at $4.5 \AA$ over a Au atom, reaches a local minimum at the midbond site $S$, and a minimum at the $H$ site (in the middle of the triangle). The

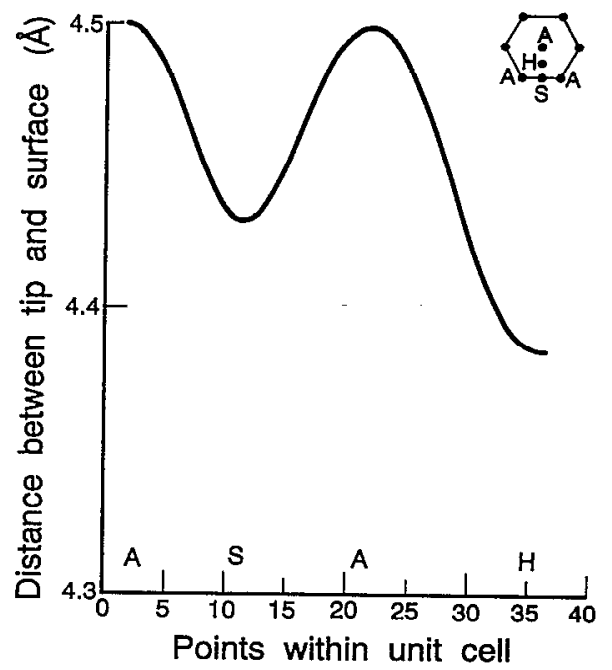

FIG. 4. Calculated scan for $\mathrm{Au}$ (111) with a tungsten tip. calculated corrugation is about $0.11 \AA$, which is in reasonable agreement with the experimental value of $0.15 \pm 0.04$ $\AA^{53}$

\section{COMPARISON WITH THE TRANSFER HAMILTONIAN EXPRESSION}

The expression for the STM current given by the transfer Hamiltonian model of Tersoff and Hamann ${ }^{5}$ is of the same form as Eqs. (14a) and (14b) or Eqs. (15a) and (15b), but with $\left|H_{n m}\left(\mathbf{k}_{s}, \mathbf{k}_{t}\right)\right|^{2}$ replaced by $\left|M_{n m}\left(\mathbf{k}_{s}, \mathbf{k}_{t}\right)\right|^{2}$, with the latter given by ${ }^{5,14,15}$

$$
M_{n m}\left(\mathbf{k}_{s} \mathbf{k}_{t}\right)=\frac{1}{2 m} \int d \mathbf{S} \cdot\left(\Psi_{n \mathrm{k}_{s}}^{*} \nabla \Psi_{m \mathbf{k}_{t}}-\Psi_{m \mathbf{k}_{t}} \nabla \Psi_{n \mathrm{k}_{s}}^{*}\right) .
$$

In Eq. (31), the integral is over any surface lying entirely within the region between the sample (left) and tip (right) electrodes, and $\Psi_{n \mathbf{k}_{s}}$ and $\Psi_{m \mathbf{k}_{t}}$ are the eigenfunctions of $H_{s}$ and $H_{t}$, respectively, where $H_{s}$ and $H_{t}$ are the model Hamiltonians defined as ${ }^{14,15}$

$$
\begin{aligned}
& H_{s}=H \text { for } z<l, \quad H_{t}=H \text { for } z>0, \\
& \text { and } H=H_{s}=H_{t} \text { for } 0<z<l .
\end{aligned}
$$

Here, $l$ is the distance between the two electrodes $(z=0$, $z=l)$ and the $z$ direction is perpendicular to the electrodes. ${ }^{15}$ For example, similar to Eq. (14b), we have

$$
\begin{aligned}
I= & 2 \pi e \sum_{n, m} \iint d \mathbf{k}_{s} d \mathbf{k}_{t}\left|M_{n m}\left(\mathbf{k}_{s}, \mathbf{k}_{t}\right)\right|^{2}\left[f\left(\epsilon_{s}\right)-f\left(\epsilon_{t}\right)\right] \\
& \times \delta\left(\epsilon_{s}-\epsilon_{t}+e v\right),
\end{aligned}
$$

where $\epsilon_{i}$ denotes $E_{i}-\mu_{i}$, with $E_{i}$ and $\mu_{i}$ being the eigenvalue of $H_{i}$ defined in Eq. (32) and the chemical potential of the $i$ electrode, respectively, $i=s, t$.

Using exponentially decaying wave functions outside of the surface of the metal and of the tip, and using a radially symmetric wave function for the tip, the integral in Eq. (31) for $M_{n m}\left(\mathbf{k}_{s}, \mathbf{k}_{t}\right)$ was evaluated by Tersoff and Hamann. In the limit of low voltage, an expression [Eq. (1) given earlier] for the current $I$ was obtained. ${ }^{5}$ Other tip models have also been employed to evaluate this matrix element, e.g., in Refs. 6-13.

Equation (19a), the expression for the STM current in the present model, can also yield the local density of states (LDOS) or Eq. (1), when one introduces approximations into Eq. (19a). We let the matrix element $\left\langle\phi_{n \mathbf{k}_{s}}|H| \phi_{t}\right\rangle$ be proportional to the overlap integral $\left\langle\phi_{n \mathbf{k}_{s}} \mid \phi_{t}\right\rangle$, the function $\phi_{r}$ being concentrated at the tip position $\mathbf{r}_{t}$, and it is next assumed that $\left\langle\phi_{n \mathbf{k}_{s}} \mid \phi_{t}\right\rangle$ is proportional to $\left\langle\phi_{n \mathbf{k}_{s}} \mid \mathbf{r}_{t}\right\rangle$, i.e., to the wave function $\phi_{n \mathbf{k}_{s}}^{*}\left(\mathbf{r}_{t}\right)$. We then have

$$
\begin{aligned}
I & \propto \sum_{n} \int d \mathbf{k}_{s}\left|\left\langle\phi_{n \mathbf{k}_{s}}|H| \phi_{t}\right\rangle\right|^{2} \delta\left(\epsilon_{s}\right) \\
& =\text { constant } \times \sum_{n} \int d \mathbf{k}_{s}\left|\phi_{n \mathbf{k}_{s}}\left(\mathbf{r}_{t}\right)\right|^{2} \delta\left(\epsilon_{s}\right) \\
& =\text { constant } \times \text { LDOS. }
\end{aligned}
$$


Thereby, the STM current in the present model then becomes proportional to the local density of states (LDOS), as in the Tersoff-Hamann model. In the actual applications, however, we shall use Eq. (19a) and compare with the approximation given by Eq. (34).

\section{CONCLUDING REMARKS}

The STM current in the present theoretical model depends on the average of the square of the sample-tip matrix elements $\left\langle\left|H_{t, n \mathbf{k}_{s}}\right|^{2}\right\rangle_{\text {av }}$ defined in Eq. (19b). The latter is, in turn, expressed in terms of the atomic coefficients in the crystal wave functions of the isolated sample and tip, and of the matrix elements between the atomic orbitals of the sample and those of the tip. In the present model, the properties such as the wave functions and constant energy surfaces of the tip and sample can be calculated using methods such as the density-functional theory and a variety of quantum chemistry methods, e.g., the tight-binding model. We employ the latter here.

As pointed out by Tersoff, most such methods using local orbitals (Gaussian type or Slater type) as a basis, though well adapted to problems of total energy and band structure, are inaccurate for the quantitative calculation of the STM current. When the sample and tip are separated by a very large distance, say $10 \AA$, in this case only, the tails of the wave functions at a large distance from the surface are important. Those local orbitals behave rather inaccurately at a large distance from the surface. On the other hand, use of the local orbitals may be justified if the sample-tip separation is such as to ensure the validity of the perturbation treatment, but not so large that the local orbitals interact strongly. When the sample-tip interaction is reasonably well treated as being between two welllocalized nearest neighboring atomic orbitals, the nearestneighbor tight-binding approximation becomes reasonable.

The present model is then applied to the graphite (0001) and $\mathrm{Au}(111)$ surfaces. It is found that the calculated corrugation for the $\mathrm{Au}(111)$ surface is in reasonble agreement with the experiment. In the case of graphite, for an experimentally relevant $W$ tip, a very small corrugation amplitude of slightly over $0.1 \AA$ is obtained, similar to the result by Tersoff and Lang, but smaller than the experimental values to date.

A number of assumptions have been employed in this treatment of STM and in its later application to adsorbates. For this reason, until calculations based on these formulas are extensively tested experimentally, any conclusions drawn from them should be treated with some reserve.

\section{ACKNOWLEDGMENTS}

It is a pleasure to acknowledge the support of this research by the Office of Naval Research, by the National Science Foundation, and by the Natural Science Research Council of Sweden.

${ }^{1}$ G. Binnig, H. Rohrer, Ch. Gerber, and E. Weibel, Phys. Rev. Lett. 49, 57 (1982); 50, 120 (1983).

${ }^{2}$ For reviews, see G. Binnig and H. Rohrer, Rev. Mod. Phys. 59, 615 (1987); P. K. Hansma and J. Tersoff, J. Appl. Phys. 61, R1 (1987); N.
D. Lang, Comments Condensed Matter. Phys. 14, 253 (1989); Scanning Tunneling Microscopy and Related Methods, edited by R. J. Behm, N. Garcia, and H. Rohrer (Kluwer, Dordrecht, 1990); M. Tsukada, K. Kobayashi, N. Isshiki, and H. Kageshima, Surf. Sci. Rep. 13, 265 (1991).

${ }^{3}$ For example, A. Martin-Rodero, F. Flores, and N. H. March, Phys. Rev. B 38, 10047 (1988); D. M. Eigler, P. S. Weiss, E. K. Schweizer, and N. D. Lang, Phys. Rev. Lett. 66, 1189 (1991).

${ }^{4}$ For example, M. J. Allen, M. Balooch, S. Subbiah, R. J. Tench, W. Siekhaus, and R. Balhorn, Scanning Microscopy 5, 625 (1991).

${ }^{5}$ J. Tersoff and D. R. Hamann, Phys. Rev. B 31, 805 (1985); Phys. Rev. Lett. 50, 1998 (1983).

${ }^{6}$ N. Garcia, C. Ocal, and F. Flores, Phys. Rev. Lett. 50, 2002 (1983).

${ }^{7}$ E. Stoll, A. Baratoff, A. Selloni, and P. Carnevali, J. Phys. C 17, 3073 (1984).

${ }^{8}$ N. D. Lang, Phys. Rev. Lett. 56, 1164 (1986); 58, 45 (1987).

${ }^{9}$ M. Tsukada and N. Shima, J. Phys. Soc. Jpn. 56, 2875 (1987).

${ }^{10}$ A. A. Lucas, H. Morawitz, G. R. Henry, J. P. Vigneron, Ph. Lambin, P. H. Cutler, and T. E. Feuchtwang, Phys. Rev. B 37, 10708 (1988).

${ }^{11}$ C. J. Chen, J. Vac. Sci. Technol. A 6, 319 (1988); 9, 44 (1991).

${ }^{12}$ E. Tekman and S. Ciraci, Phys. Rev. B 40, 10286 (1989).

${ }^{13}$ W. Sacks and C. Noguera, Phys. Rev. B 43, 11612 (1991).

${ }^{14}$ I. Bardeen, Phys. Rev. Lett. 6, 57 (1961).

${ }^{15}$ For a review, see C. B. Duke, Solid State Physics, edited by F. Seitz and D. Turnbull (Academic, New York, 1969), Suppl. 10.

${ }^{16}$ A. Selloni, P. Carnevali, E. Tosatti, and C. D. Chen, Phys. Rev. B 31, 2602 (1985).

${ }^{17}$ R. M. Tromp, R. J. Hamers, and J. E. Demuth, Phys. Rev. B 34, 1388 (1986).

${ }^{18}$ For a review, see J. Tersoff, in Scanning Tunneling Microscopy and Related Methods, edited by R. J. Behm, N. Garcia, and H. Rohrer, (Kluwer, Dordrecht, 1990).

${ }^{19}$ J. Tersoff and N. D. Lang, Phys. Rev. Lett. 65, 1132 (1990).

${ }^{20}$ D. Lawunmi and M. C. Payne, J. Phys. Condensed Matter 2, 3811 (1990).

${ }^{21}$ C. J. Chen, Phys. Rev. Lett. 65, 448 (1990).

${ }^{22}$ E. Tekman and S. Ciraci, Phys. Rev. B 42, 1860 (1990).

${ }^{23}$ P. Hohenberg and W. Kohn, Phys. Rev. 136, B864 (1964).

${ }^{24}$ W. Kohn and L. J. Sham, Phys. Rev. 140, A1133 (1965); cf. N. D. Lang and W. Kohn, Phys. Rev. B 1, 4555 (1970).

${ }^{25}$ M. Levy, Proc. Natl. Acad. Sci. USA 76, 6062 (1979).

${ }^{26} \mathrm{~A}$. review is given in R. G. Parr and W. Yang, Density-Functional Theory of Atoms and Molecules (Oxford, New York, 1989).

${ }^{27}$ J. C. Slater, Phys. Rev. 81, 385 (1951).

${ }^{28}$ For a review, see J. C. Slater, The Self-Consistent Field for Molecules and Solids Quantum Theory of Molecules and Solids (McGraw-Hill, New York, 1974), Vol. 4.

${ }^{29}$ For example, J. C. Slater and G. F. Koster, Phys. Rev. 94, 1498 (1954); N. W. Ashcroft and N. D. Mermin, Solid State Physics (Holt, Rinehart, and Winston, New York, 1976), Chap. 10.

${ }^{30} \mathrm{~A}$ recent review of applications of the tight-binding method to chemical problems is given in R. Hoffmann, Solids and Surfaces: $A$ Chemist's View of Bonding in Extended Structures (VCH, New York, 1988).

${ }^{31}$ J. R. Oppenheimer, Phys. Rev. 31, 66 (1928).

${ }^{32}$ R. Hoffmann, J. Chem. Phys. 39, 1397 (1963).

${ }^{33}$ R. A. Marcus, J. Chem. Phys. 98, 5604 (1993).

${ }^{34}$ The first-order perturbation problem in the presence of two Hamiltonians was first treated by Oppenheimer in the calculation of the field ionization of atomic hydrogen (Refs. 15 and 31). In Oppenheimer's case, the two Hamiltonians are the Hamiltonian for the free hydrogen atom $H_{1}=-[1 /(2 m)] \nabla^{2}-\left(e^{2} / r\right)$, and that for an electron in a uniform electric field $H_{2}=-[1 /(2 m)] \nabla^{2}-e E_{z}$, where $E_{z}$ is the applied electric ficld whose direction is the $z$ axis, and where both Hamiltonians are in units of $\hbar=1$. The total Hamiltonian is written as $H=-[1 /(2 m)] \nabla^{2}$ $-\left(e^{2} / r\right)-e E_{z} z=H_{1}+H_{2}+[1 /(2 m)] \nabla^{2}$. Compared to the Oppenheimer's Hamiltonian, the Hamiltonian in Eq. (3) contains an extra term $v_{s-t}$ the sample-tip interaction potential which is important for an electron tunneling from the tip to the sample or vice versa.

${ }^{35}$ The analogous many-electron case for electron transfer with only two electronic states is discussed in N. R. Kestner, J. Logan, and J. Jortner, J. Phys. Chem. 78, 2148 (1974). In the present treatment, an infinite number (other than two) electronic states are involved. A review of electron transfer is given in R. A. Marcus and N. Sutin, Biochim. Biophys. Acta 811, 265 (1985). 
${ }^{36}$ Suppose that in absence of an applied voltage (potential) $\mu_{i}$ is denoted by $\mu_{i}^{0}, i=s, t$, and so $\mu_{s}^{0}=\mu_{t}^{0}$. When a voltage $v\left(=v_{t}-v_{s}\right)$ is applied between tip and sample, $\mu_{i}=\mu_{i}^{0}+e v_{i}$ and so $\mu_{s}-\mu_{t}=\mu_{s}^{0}-\mu_{t}^{0}-e\left(v_{t}-v_{s}\right)$ $=-e v$. Equation (12) was obtained in this way.

${ }^{37}$ For example, H. Ou-Yang, B. Källebring, and R. A. Marcus, J. Chem. Phys. 98, 7405 (1993); and references cited therein.

${ }^{38}$ The system of a semi-infinite linear chain of transition atoms belongs to the $C_{\infty \cup}$ group. The coupling matrix elements $H_{m m^{\prime}}^{j j^{\prime}} \equiv\left\langle d_{m}^{j}|H| d_{m^{\prime}}^{j^{\prime}}\right\rangle \neq 0$ only if $d_{m}^{j}$ and $d_{m}^{i^{\prime}}$, belong to the same irreducible representation of the molecular point group (Ref. 56). In the Character Table of $C_{\infty}, d_{z^{2}}$ belongs to irreducible representation $A_{1}, d_{x z}$, and $d_{y z}$ to $E_{1}$, and $d_{x^{2}-y^{2}}$ and $d_{x y}$ to $E_{2}$, respectively (Ref. 56). Therefore, only two matrix elements $H_{\left(x^{2}-y^{2}\right)(x y)}^{j j^{\prime}}$ and $H_{(x z)(y z)}^{j j^{\prime}}$ may be possibly nonzero. However, because of the choice of orbitals they vanish also. A symmetry operation $\sigma_{v}$ (reflection in the $x z$ plane) leaves $H$ invariant, but causes $x_{j} \rightarrow x_{j}$, $y_{j} \rightarrow-y_{j}, z_{j} \rightarrow z_{j}$. Thereby, $d_{x^{2}-y^{2}}^{j} \rightarrow d_{x^{2}-y^{2}}^{j}$ and $d_{x y}^{j} \rightarrow-d_{x y}^{j}$ under this operation, and so the matrix element $H_{\left(x^{2}-y^{2}\right)(x y)}^{j j^{\prime}}$ vanishes. Similarly, it is found that $H_{(x z)(y z)}^{j j^{\prime}}=0$.

${ }^{39}$ G. Binnig, H. Fuchs, Ch. Gerber, H. Rohrer, E. Stoll, and E. Tosatti, Europhys. Lett. 1, 31 (1985).

${ }^{40}$ J. M. Soler, A. M. Baro, N. Garcia, and H. Rohrer, Phys. Rev. Lett. 57, 444 (1986); J. B. Pethica, ibid. 57, 3235 (1986).

${ }^{41}$ T. Iri, H. Shiba, and H. Nishikawa, Jpn. J. Appl. Phys. 31, 1441 (1992).

${ }^{42}$ Y. Sugawara, T. Ishizaka, and S. Morita, Jpn. J. Appl. Phys. 29, 1533 (1990).

${ }^{43}$ I. P. Batra, N. Garcia, H. Rohrer, H. Salemink, E. Stoll, and S. Ciraci, Surf. Sci. 181, 126 (1987).

${ }^{44}$ J. Tersoff, Phys. Rev. Lett. 57, 440 (1986).

${ }^{45}$ H. J. Mamin, E. Ganz, D. W. Abraham, R. E. Thomson, and J. Clarke, Phys. Rev. B 34, 9015 (1986).
${ }^{46}$ D. Tomanek, S. G. Louie, H. J. Mamin, D. W. Abraham, E. Ganz, R. E. Thomson, and J. Clarke, Phys. Rev. B 35, 7790 (1987); D. Tomanek and S. G. Louie, ibid. 37, 8327 (1988).

${ }^{47} \mathrm{~A}$ discussion of STM of graphite using a two-layer description is given by M. R. Soto, J. Microsc. 152, 779 (1988); Surf. Sci. 225, 190 (1990).

${ }^{48} \mathrm{~A}$ review of the theoretical treatment of graphite using cluster tips is given by M. Tsukada et al. in Ref. 2.

${ }^{49}$ See, e.g., N. W. Ashcroft and N. D. Mermin in Ref. 29.

${ }^{50}$ Within the nearest neighbor form of the tight-binding approximation, Eq. (25) for $\phi_{n \mathbf{k}_{s}}$ is the solution of the difference equations for the semi-infinite graphite with a $(0001)$ surface in which four bands are considered. The difference equations can be reduced to four linear equations. The coefficients $A_{n g}$ are obtained by solving the linear equations along with the normalization condition.

${ }^{51}$ J. D. Bernal, Proc. R. Soc. London, Ser. A 160, 749 (1924).

${ }^{52} \mathrm{~A}$ different treatment has also been reported by $\mathrm{J}$. Tersoff (Ref. 44 ) and by A. L. Tchougreeff and R. Hoffmann [J. Phys. Chem. 96, 8993 (1992)], respectively. For a monolayer of graphite, they proposed a charge-density state explanation of the asymmetry of the STM images of graphite, namely, that only three of the six atoms in each carbon hexagon are visible.

${ }^{53} \mathrm{Ch}$. Woll, S. Chiang, R. J. Wilson, and P. H. Lippel, Phys. Rev. B 39, 7988 (1989); V. M. Hallmark, S. Chiang, J. F. Rabolt, J. D. Swalen, and R. J. Wilson, Phys. Rev. Lett. 59, 2879 (1987).

${ }^{54}$ J. Wintterlin, J. Wiechers, H. Burne, T. Gritsch, H. Höfer, and R. J. Behm, Phys. Rev. Lett. 62, 59 (1989).

${ }^{55}$ See, e.g., N. W. Ashcroft and N. D. Mermin in Ref. 29, p. 289, and also B. Källebring, H. Ou-Yang, and R. A. Marcus, J. Chem. Phys. (unpublished).

${ }^{56}$ For example, F. A. Cotton, Chemical Applications of Group Theory (Wiley, New York, 1990). 